\title{
Design and implementation of a neural network controller for real-time adaptive voltage regulation
}

\author{
Xiao-Hua Yu, Weiming Li, Taufik \\ Department of Electrical Engineering, California Polytechnic State University, San Luis Obispo, CA 93407, USA
}

\begin{abstract}
A B S T R A C T
An adaptive controller based on multi-layer feed-forward neural network is developed for real-time voltage regulation of a class of PSFB (phase-shifted full-bridge) DC-DC converters. The controller has the unique advantages of nonlinear mapping and adaptive learning, and performs well over a wide range of input voltages and output load currents. The controller is implemented and tested in hardware using a DSP (digital signal processor) board. Experimental results show that it outperforms conventional controllers in both line regulation and load regulation.
\end{abstract}

Keywords:

Neural network controller, DC-DC voltage converter, Neural network hardware

\section{Introduction}

A DC-DC converter is an integral part of many electrical devices, especially in portable electronic equipment that requires low-voltage/low-power operation and high reliability. Pulsewidth modulation (PWM) is often employed to control the DC output voltage by modulating the duty cycle via electronic switching circuits. To improve the power efficiency, many different switching circuit topologies $[2,3,5,6,10,16]$ have been proposed, including phase-shifted zero-voltage switching fullbridge (PSFB) converters. Unlike other topologies which may require additional active circuit components or variable operating frequencies, PSFB employs a soft-switching technology called zero-voltage-switching (ZVS) that is especially suitable for the advanced MOSFETs (metal oxide semiconductor field-effect transistors) implementation [5,6,16].

To keep the output of a DC-DC converter stable, an appropriate control signal should be applied. In conventional controller design, it is assumed that all the circuit components are ideal with no performance degradation and power loss; and the circuit is operated at a stable bias point so that it can be modeled by a set of linear equations [4,17]. However, in practice, the switching network is highly nonlinear and an accurate mathematical model is very difficult to obtain. In addition, the supply voltage and load current may also fluctuate over a wide range. Thus, real-time adaptive control becomes necessary to achieve proper system performance.

Recently, artificial neural networks (ANN) have been applied to improve the performance of DC-DC converters to dynamical system changes $[8,15]$. However, no prior work has yet been reported to control a PSFB converter using a neural network approach.

In this paper, a controller design employing a multi-layer feedforward neural network is investigated. A Matlab Simulink ${ }^{1 \mathrm{~B}}$ model is developed to generate the data set; and the neural network is trained off-line using a back-propagation algorithm. A network pruning algorithm is also applied to determine the appropriate size of the controller. Finally, the proposed neural network controller for a PSFB DC-DC converter is implemented on a DSP (digital signal processor) evaluation board. To accommodate variations in system dynamics and parameters while maintaining fast response, an on-line training routine is included to fine-tune the weights of the neural network controller in real-time. Satisfactory experimental results are obtained to show that this neural network controller can provide improved performance over conventional controllers in both line regulation and load regulation.

\section{Modeling and simulation}

\subsection{The neural network controller for PSFB DC-DC converter}

The conventional approach to control the output voltage of a DC-DC converter is to modulate the duty cycle of gate control 


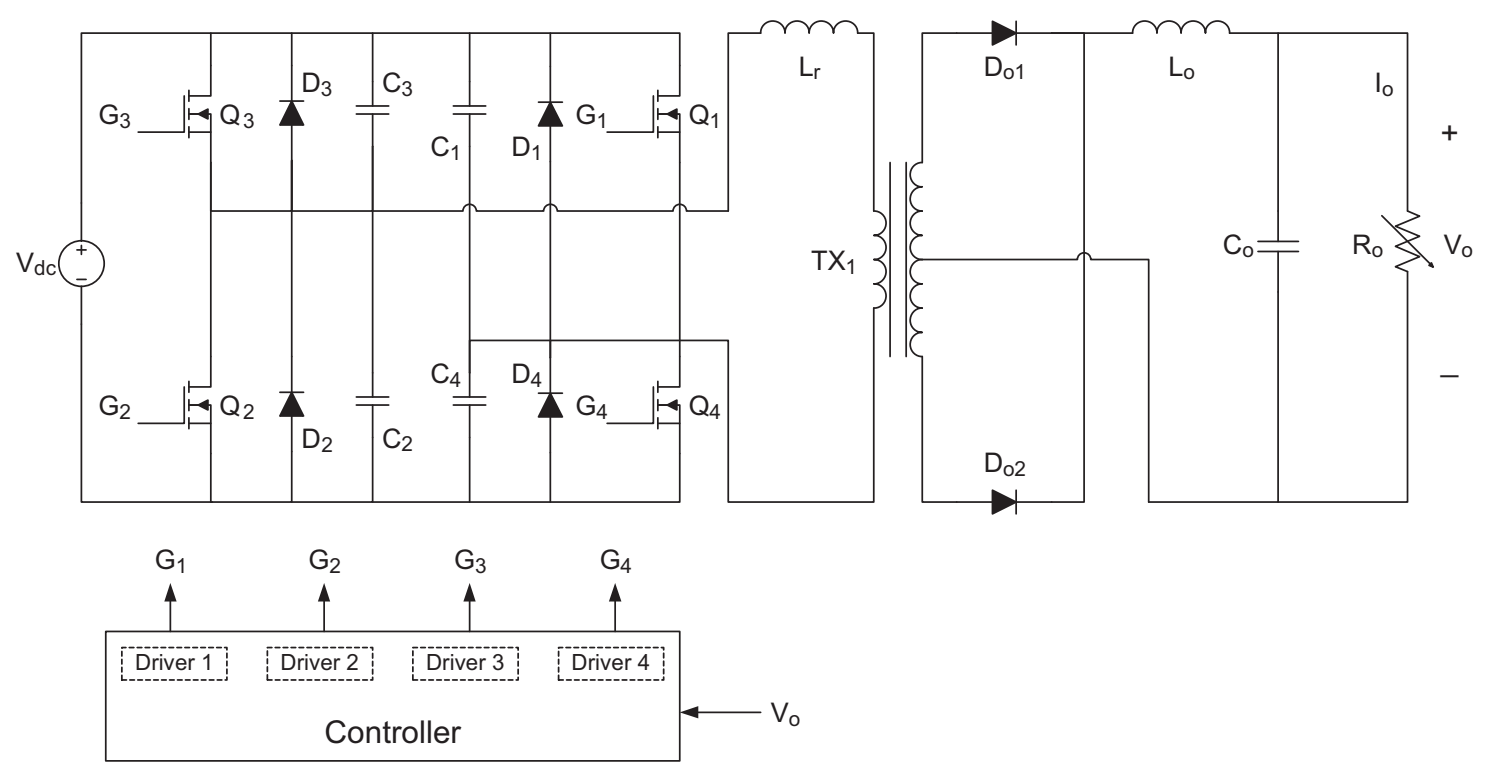

Fig. 1. Basic configuration of a typical PSFB DC-DC converter.

signals (i.e., the ratio of time when the signal is at the "high" level versus its period) in a switching network. The basic configuration of a typical PSFB DC-DC converter is shown in Fig. 1. The circuit consists of four power switches $\left(Q_{1}, Q_{2}, Q_{3}\right.$, and $\left.Q_{4}\right)$, a transformer $\left(T X_{1}\right)$, and an output stage. The power switches act as the gate for currents to flow from the source $V_{d c}$ to the load. The timing signals for each switch to turn on and off are provided by the driver circuit. The high frequency transformer $\left(T X_{1}\right)$ provides isolation and steps down the input voltage to slightly above an appropriate output voltage level. Note that there is a small inductor $\left(L_{r}\right)$ connected in series with the transformer to ensure proper resonant frequency for the soft switching. The output stage consists of two diodes $\left(D_{01}\right.$ and $\left.D_{02}\right)$ to convert AC pulse signals out of the transformer into DC pulses. The LC filter $\left(L_{0}\right.$ and $\left.C_{0}\right)$ cleans up the DC signal further to yield a DC voltage with acceptable ripple at the load. Based on circuit analysis, the duty cycle and timing scheme to drive the four switching MOSFETs $\left(Q_{1}\right.$, $Q_{2}, Q_{3}$, and $Q_{4}$ ) is highly nonlinear [1,16] and leads to a very complicated controller design using conventional approaches.

In this research, a multi-layer feed-forward artificial neural network is employed to achieve real-time adaptive control. Since the duty cycle is a nonlinear function of input voltage $V_{\text {in }}$ and load current $I_{0}[11,12]$, they are chosen to be the inputs of the neural network. In practice, $V_{\text {in }}$ and $I_{0}$ may fluctuate over a wide range. The converter output voltage should be kept at a constant; otherwise, the control signal should be changed and a different duty cycle should be applied. Thus, we choose the output voltage change as another input to the neural network. The output of the neural network controller is the duty cycle $\Phi$, a positive real number bounded between 0 and 1 :

$0 \leq \Phi \leq 1$

The nonlinear sigmoid function is chosen as the activation function for each neuron:

$f(x)=\frac{1}{1+e^{-a x}}$

where $a$ is a constant parameter $(a>0)$. Fig. 2 shows an inputoutput diagram of the neural network controller.

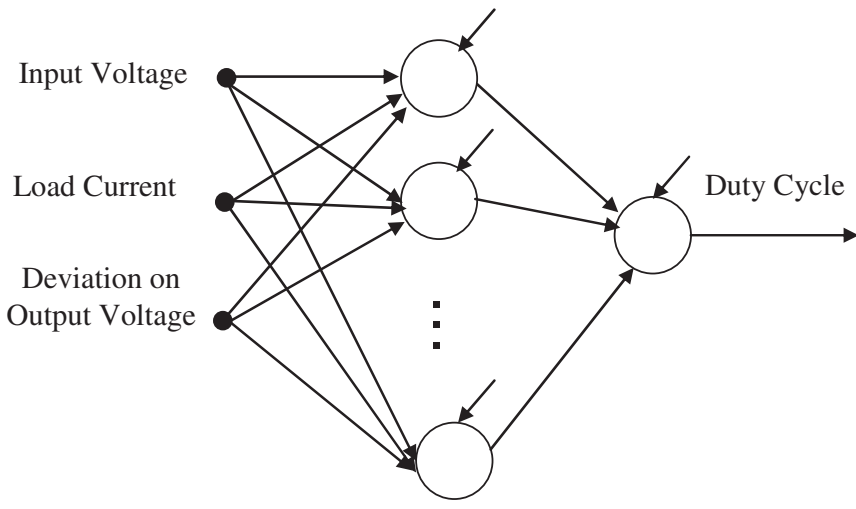

Fig. 2. The neural network controller.

\subsection{The neural network pruning algorithm}

It is well known that a multi-layer feed-forward neural network of an appropriate size can approximate any nonlinear function to a desired accuracy after it is fully trained $[7,14]$. However, before such a neural network can be employed, an optimal configuration (i.e., number of layers, number of neurons in each layer) should be pre-determined, especially in the realtime environment where speed is crucial.

In this research, a sensitivity based neural network pruning approach $[9,13,18]$ is employed to determine an optimal neural network controller configuration. Sensitivity is a performance measure which indicates the contribution of each individual weight/node to the overall neural network performance. The sensitivity of a global error function $J(\cdot)$ with respect to each weight, $s_{i j}$, can be defined as the following [13]:

$s_{i j}=J\left(w_{i j}=0\right)-J\left(w_{i j}=w_{i j}^{f}\right)=J\left(\right.$ without $\left.w_{i j}\right)-J\left(\right.$ with $\left.w_{i j}\right)$

where $w_{i j}$ is the weight/connection of the neural network from node $i$ to $j$; $w_{i j}^{f}$ is the final value of weight after training; and $J(\cdot)$ is the RMSE (root mean square error) of NN output. In [9], it is shown that for the back-propagation algorithm, Eq. (3) can be 
approximated by

$\hat{s}_{i j}=\sum_{n=1}^{N}\left[\Delta w_{i j}(n)\right]^{2} \frac{w_{i j}^{f}}{\eta\left(w_{i j}^{f}-w_{i j}^{i}\right)}$

where $\eta$ is the learning rate (usually in the range between 0 and 1. In this simulation, $\eta$ is chosen to be 0.5$) ; \Delta w_{i j}$ is the weight update; and $N$ is either the number of training patterns for each $\mathrm{NN}$ weight update (if bench training is employed), or $N=1$ (for online training).

Once a neural network is trained to achieve the input/output mapping with desired accuracy, the sensitivity calculation based on Eq. (4) can be activated. If the sensitivity of a particular weight is smaller than a pre-set threshold, this weight is insignificant and can be deleted. Furthermore, a neuron can be removed when the sensitivities of all the weights related with this neuron are below the threshold:

$w_{i j}= \begin{cases}0 & \text { if }\left|\hat{s}_{i j}\right|<\tau \\ w_{i j} & \text { if }\left|\hat{s}_{i j}\right| \geq \tau\end{cases}$

A Matlab Simulink ${ }^{\circledR}$ model is developed to train the neural network off-line [11]. To determine the optimal size for the neural network controller, we start with a three layer feed-forward neural network which has one hidden layer with 10 neurons. The network weights are initialized at random with uniform distribution over the interval $[-1,1]$. The total number of weights is 51 , including the bias term of each hidden neuron and the output neuron. The training performance is shown in Fig. 3, which illustrates that the RMSE decreases with training epochs. After 500 training cycles (using the back-propagation algorithm), an RMSE of 0.0119 is obtained.

The network pruning algorithm is applied to reduce the size of neural network. The choice of the threshold value for sensitivity is generally application dependent and based on a trial-and-error process. The sensitivity value used in this simulation is 0.03 for all the weights, which is also the median of all the sensitivities of the network. After removing 22 weights with smaller sensitivities, the RMSE of the reduced network output is increased to 0.0257. To improve the performance of the pruned neural network, training is continued further. After another 500 training epochs, the RMSE is reduced to 0.0096, as shown in Fig. 4. Note that the neural network dimension is reduced dramatically by $43.14 \%$ (from 51 to 29 weights) while still maintaining similar performance.

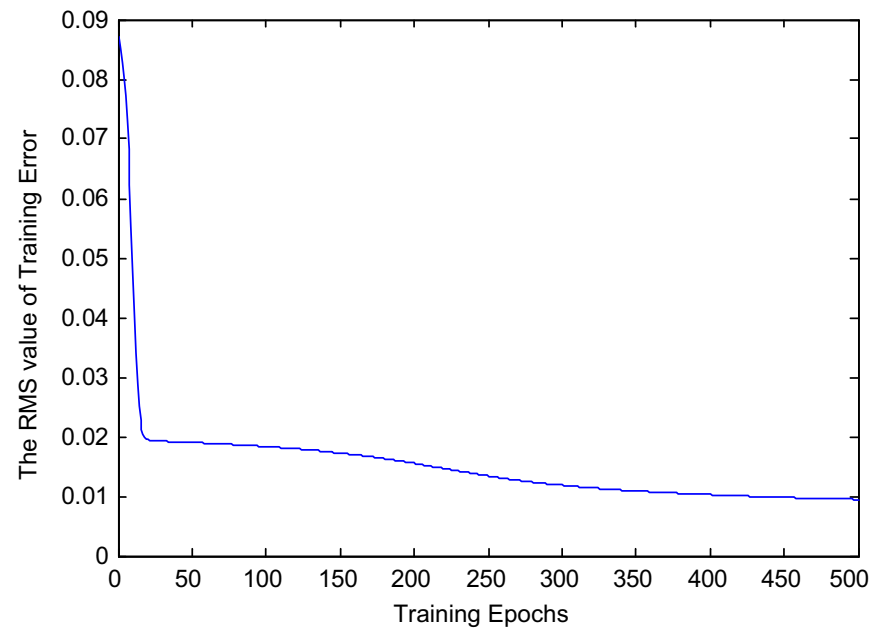

Fig. 3. The RMS of output error vs. training epochs.

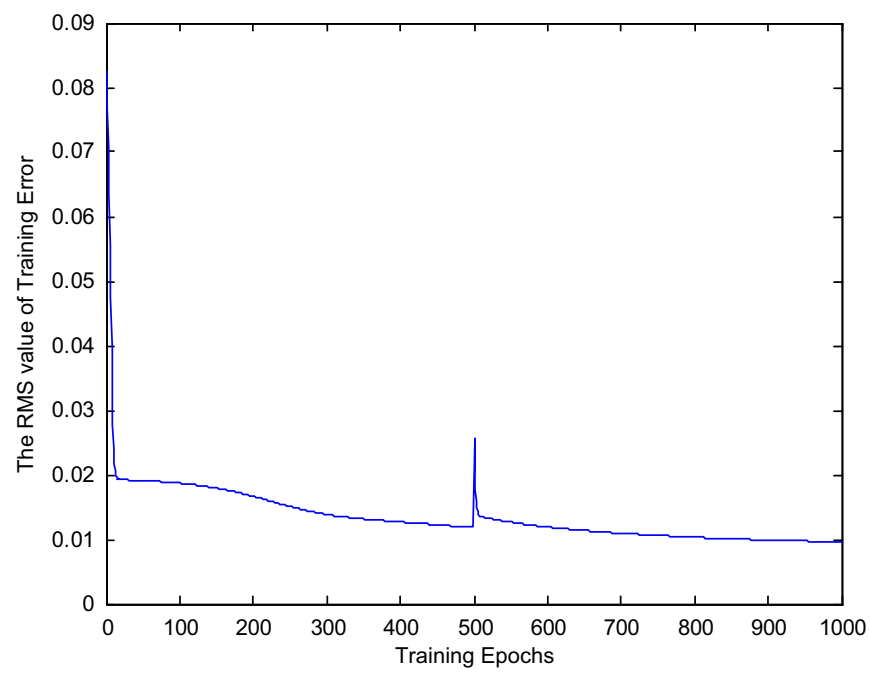

Fig. 4. The RMS of output error vs. training epochs.

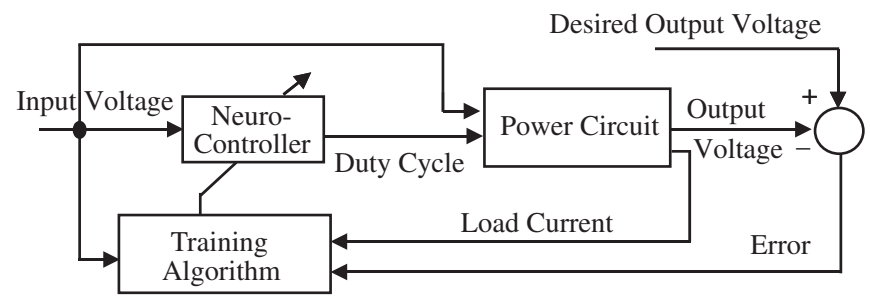

Fig. 5. The system block diagram.

\section{Experimental results}

The proposed neural network controller is implemented on a Texas Instrument ${ }^{\circledR}$ DSP (digital signal processor) evaluation board (eZdsp ${ }^{\mathbb{R}}$ F2812) to control a commercially available phase-shifted zero-voltage-switching DC-DC converter module (UCC3895EVM001 ). The on-board digital signal processor TMS320F2812 is a 32bit CPU with 150 MIPS (million instructions per second) operating speed; and the neural controller is programmed in $C$ language. The PSFB DC-DC converter board has a nominal input voltage of $48 \mathrm{~V}$ and a nominal output voltage of $3.3 \mathrm{~V}$, with a built-in conventional analog controller (UCC3895).

The overall system block diagram is shown in Fig. 5. To test the performance of neural network controller, the on-board conventional controller (UCC3895) is removed and replaced with the neuro-controller. The input voltage, output voltage, and load current of the DC-DC converter are connected to the ADC (analog to digital converter) input channels of the DSP module. Note that the power circuit is driven by $400 \mathrm{KHz}$ PWM signals; thus the raw output voltage signal can be quite noisy, especially during the switch turn-on and turn-off time. To reduce switching noise, multiple samples are taken to obtain the time averaged inputs. The neural network controller output is connected to the power circuit through the DAC (digital to analog converter) output channel of the DSP board and the jumpers on the power circuit module. Because the neural network is trained off-line (see Section 2), the converter output voltage is systematically lower than the desired value. This is due to our ideal Simulink model 
which does not take into account parasitic voltage drops and power consumptions, as well as the value variations of circuit components. Therefore, on-line training is implemented to finetune the weights of the neural network. With this correction, the performance of the neural network controller is significantly improved.

In the back-propagation algorithm, the gradient of the output error function with respect to each weight should be calculated. In the DC-DC converter, PWM (pulse-width modulation) is employed to control the output voltage by adjusting the duty cycle of square waves. Note that the duty cycle, i.e., the output of the neural network controller, is not monitored directly from the circuit; one only monitors the output voltage of the DC converter. And in fact, our control objective is to keep the output stable under various input voltage and load current conditions. That is, the following objective function is minimized:

$E=\frac{1}{N} \sum_{n=1}^{N}\left[V_{0}(n)-V_{0}^{N N}(n)\right]^{2}$

where $V_{0}(n)$ \&epresent the desired output of the DC converter, $V_{0}^{N N}(n)$ represents the output voltage with the neural network controller.

To design the neural network controller more efficiently, the difference between the current output voltage of the power circuit and the desired value is monitored and compared with a preset threshold. The control signal (i.e., duty cycle) is unchanged if this deviation is less than the threshold. When it is larger than the threshold, the neural network controller is activated and a new duty cycle is applied to the circuit. If the DC-DC converter output is still not satisfactory, then on-line training is performed and the neural network weights are adjusted to minimize the voltage deviation.

The neural network controller is tested over a range of different input voltages with full load, as shown in Fig. 6, where the $y$-axis represents the deviation of the output voltage (with respect to the nominal value) and the $x$-axis represents the input voltage. The ability of a power circuit to maintain a constant output voltage under various input conditions is called line regulation. In Fig. 6, the solid line represents the voltage deviation with neural network controller while the dashed line represents the results using the on-board built-in analog controller. For most cases, the output voltage controlled by the neural network is more stable than the one controlled by the conventional controller; in other words, the neural network controller achieves better line regulation, especially at lower input voltages.

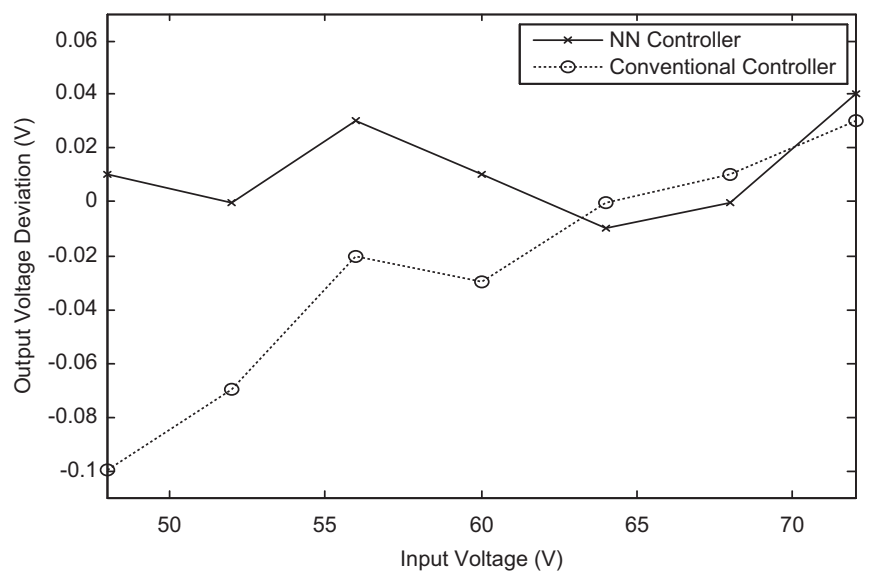

Fig. 6. Output voltage deviation vs. input voltage (at load current 15 A).

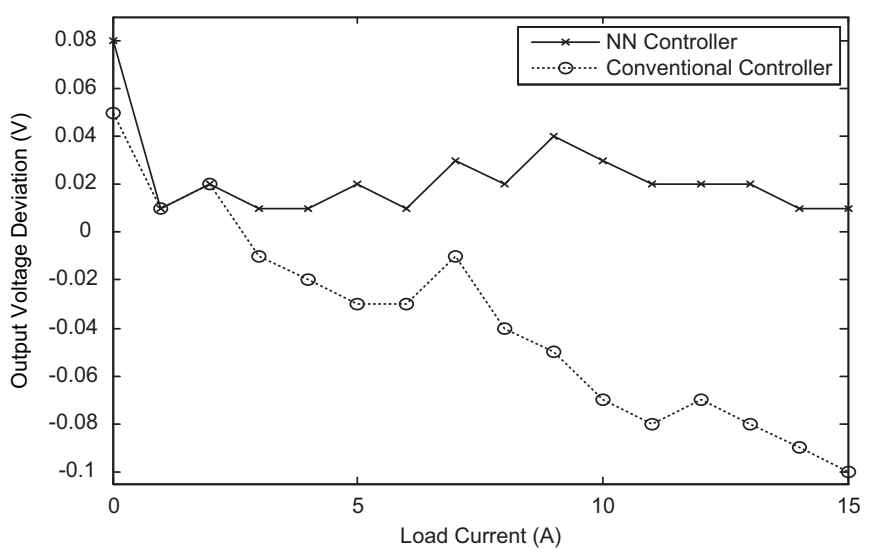

Fig. 7. Output voltage deviation vs. load current (at nominal input).

Load regulation is a measure of the ability of the power circuit to maintain its output constant when the load current changes. The experimental results are shown in Fig. 7, where the $y$-axis represents the deviation of the output voltage (with respect to the nominal value) and the $x$-axis represents the load current. Similar to Fig. 6, the solid line represents the voltage deviation with neural network controller while the dashed line represents the results with the conventional controller. The input voltage is at $48 \mathrm{~V}$ (nominal value). The neural network controller also achieves better load regulation, especially at high load currents.

\section{Conclusions}

A neural network controller for a class of PSFB DC-DC converters is designed, implemented and tested on a digital signal processor evaluation board. To determine the appropriate dimension of this neural network controller, sensitivity analysis is applied and a network pruning algorithm is employed. Our experimental results show that real-time adaptive control is achieved under various input and load conditions; and the neural network controller outperforms a conventional controller in terms of both line regulation and load regulation. More research works will be performed to optimize the software to further speed up the response of neural network controller.

\section{References}

[1] B. Andreycak, Designing a phase shifted zero voltage transition (ZVT) power converter, Unitrode Power Supply Design Seminar Manual, SEM-900, 1993, pp. $1-15$.

[2] J.M. Carrasco, E. Galván, G.E. Valderrama, R. Ortega, A. Stankovic, Analysis and experimentation of nonlinear adaptive controllers for the series resonant converter, IEEE Transactions on Power Electronics 15 (3) (2000) 536-544.

[3] P.R. Chetty, Resonant power supplies: their history and status, IEEE Aerospace and Electronic Systems Magazine 7 (4) (1992) 23-29.

[4] B. Choi, J. Kim, B.H. Cho, S. Choi, C.M. Wildrick, Designing control loop for DCto-DC converters loaded with unknown AC dynamics, IEEE Transactions on Industrial Electronics 49 (4) (2002) 925-932.

[5] H.-S. Choi, B.H. Cho, Novel zero-current-switching (ZCS) PWM switch cell minimizing additional conduction loss, IEEE Transactions on Industrial Electronics 49 (1) (2002) 165-172.

[6] H.-S. Choi, J.-W. Kim, B.H. Cho, Novel zero-voltage and zero-current-switching (ZVZCS) full-bridge PWM converter using coupled output inductor, IEEE Transactions on Power Electronics 17 (5) (2002) 641-648.

[7] S. Haykin, Neural Networks: A Comprehensive Foundation, Prentice-Hall, Englewood Cliffs, NJ, 1999. 
[8] F. Kamran, R.G. Harley, B. Burton, T.G. Habetler, M.A. Brooke, A fast on-line neural-network training algorithm for a rectifier regulator, IEEE Transactions on Power Electronics 13 (2) (1998) 366-371.

[9] E. Karnin, A simple procedure for pruning back-propagation trained neural networks, IEEE Transactions on Neural Networks 1 (2) (1990) 239-242.

[10] M.G. Kim, M.J. Youn, An energy feedback control of series resonant converters, IEEE Transactions on Power Electronics 6 (3) (1991) 338-345.

[11] W. Li, X.-H. Yu, Improving DC power supply efficiency with neural network controller, in: Proceedings of the IEEE International Conference on Contro and Automation, May 2007, pp. 1575-1580.

[12] W. Li, X.-H. Yu, A self-tuning controller for real-time voltage regulation, in: Proceedings of the IEEE International Joint Conference on Neural Networks, August 2007, pp. 2010-2014

[13] M. Mozer, P. Smolensky, Skeletonization: a technique for trimming the fat from a network via relevance assessment, in: D.S. Touretzky (Ed.), Advances in Neural Information Processing, Morgan Kaufman, Los Altos, CA, 1989, pp. 107-115.

[14] K. Narendra, et al., Identification and control of dynamical systems using neural networks, IEEE Transactions on Neural Networks 1 (1) (1990) 4-27.

[15] J.M. Quero, J.M. Carrasco, L.G. Franquelo, Implementation of a neural controller for the series resonant converter, IEEE Transactions on Industrial Electronics 49 (3) (2002) 628-639.

[16] X. Ruan, Y. Yan, A novel zero-voltage and zero-current-switching PWM fullbridge converter using two diodes in series with the lagging leg, IEEE Transactions on Industrial Electronics 48 (4) (2001) 777-785.

[17] Texas Instrument Inc., BiCMOS advanced phase shift PWM controller, Data sheet of UCC3895, 2001.

[18] X.-H. Yu, Adaptive neural network structure based on sensitivity analysis, in: Proceedings of the World Forum on Smart Materials and Smart Structures Technology, Taylor \& Francis, May 2007, pp. 448.

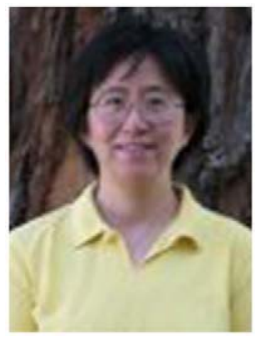

Xiao-Hua (Helen) Yu received her Ph.D. in Electrical and Computer Engineering from University of California, Irvine, in 1998. She is now an Associate Professor in the Department of Electrical Engineering, California Polytechnic State University, San Luis Obispo. Her current research interests include computational intelligence, stochastic control, and digital signal processing.

Weiming Li received his M.S. degree in Electrical Engineering from California Polytechnic State University, San Luis Obispo, in 2006. He is currently an Electrica Engineer at St. Jude Medical, Sylmar, CA.

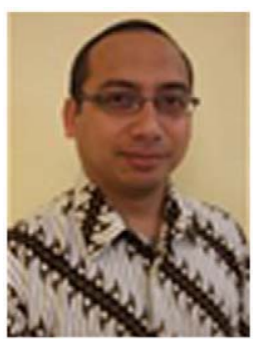

Taufik was born in Jakarta, Indonesia. He received the B.S. degree in electrical engineering from the Northern Arizona University, Flagstaff, in 1990, the M.S. degree in electrical engineering from the University of Illinois, Chicago, in 1993, and the Doctor of Engineering degree from Cleveland State University, Cleveland, $\mathrm{OH}$, in 1999. He is currently a Professor in the Electrical Engineering Department, California Polytechnic State University, San Luis Obispo. His current research interests include the area of switched mode DC-DC converters. 